\section{$\varepsilon_{0}$}

${ }^{1}$ Department of Thoracic Surgery, Birmingham Heartlands Hospital,

Birmingham, UK

${ }^{2}$ Department of Radiology, City Hospital, Birmingham, UK ${ }^{3}$ Department of

Histopathology, Birmingham Heartlands Hospital,

Birmingham, UK

\section{Correspondence to}

Maninder S Kalkat,

Birmingham Heartlands

Hospital, Bordesley Green East, Birmingham B9 5SS, UK;

maninder.kalkat@

heartofengland.nhs.uk

Received 22 June 2016 Accepted 18 July 2016

Published Online First

5 September 2016

\title{
Thoracic splenosis masquerading as advanced lung cancer
}

\author{
Mohammedabbas Remtulla, ${ }^{1}$ Nigel E Drury, ${ }_{1}^{1}$ Nazia A Kaushal, ${ }^{2}$ Simon E Trotter, ${ }^{3}$ \\ Maninder S Kalkat ${ }^{1}$
}

A 38-year-old man presented with a history of influenza-like symptoms and a persistent dry cough. He had been previously fit and well, other than a road traffic collision 20 years ago, in which he sustained bilateral pneumothoraces, left hemidiaphragm injury with herniation of the stomach into the left hemithorax and splenic injury. $\mathrm{He}$ therefore had undergone an emergency laparotomy, splenectomy and repair of the left hemidiaphragm. He was a non-smoker and denied contact with TB or asbestos. Physical examination was unremarkable other than a laparotomy scar.
Chest radiograph showed a large opacity behind the cardiac silhouette suspicious for primary lung cancer (figure 1A). CT of the thorax and abdomen confirmed a $41 \mathrm{~mm}$ soft tissue mass in the left lower lobe, closely related to the posterior aspect of the left ventricle with no clear fat plane, which was concerning for local invasion (figure 1B). There were multiple other pleural-based nodules in the lower left hemithorax, measuring up to $21 \mathrm{~mm}$; no right-sided pulmonary lesions or mediastinal lymphadenopathy were seen. The spleen was absent but further nodules were noted posterior to

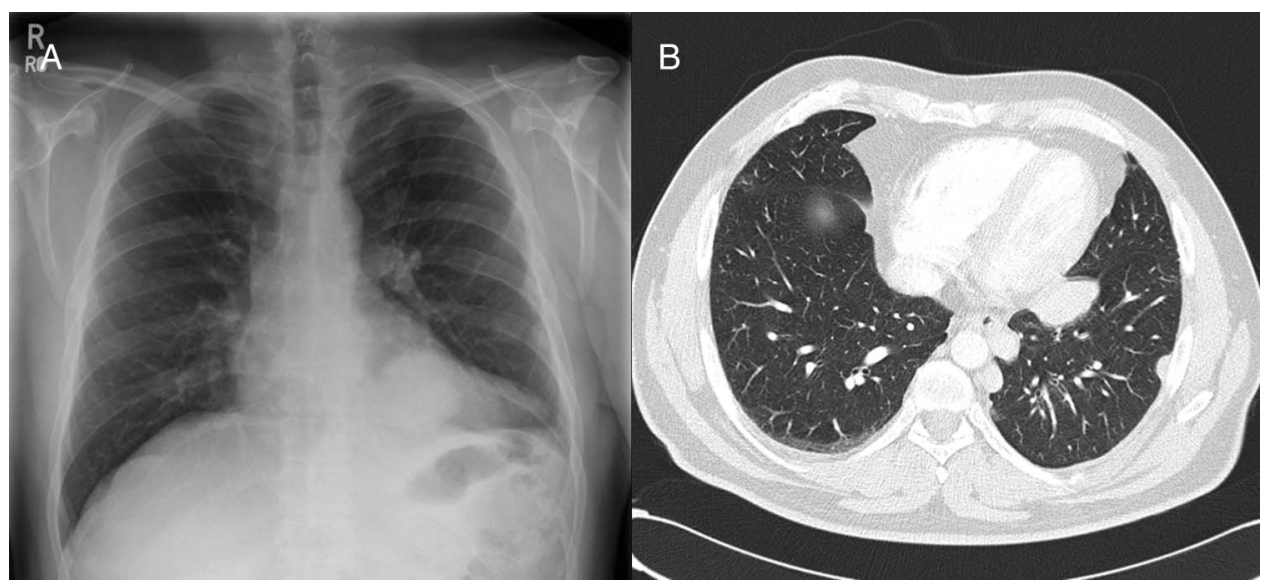

Figure 1 Chest radiograph showing a large mass behind the cardiac silhouette (A) and CT demonstrating multiple soft tissue pleural nodules in the left hemithorax (B).
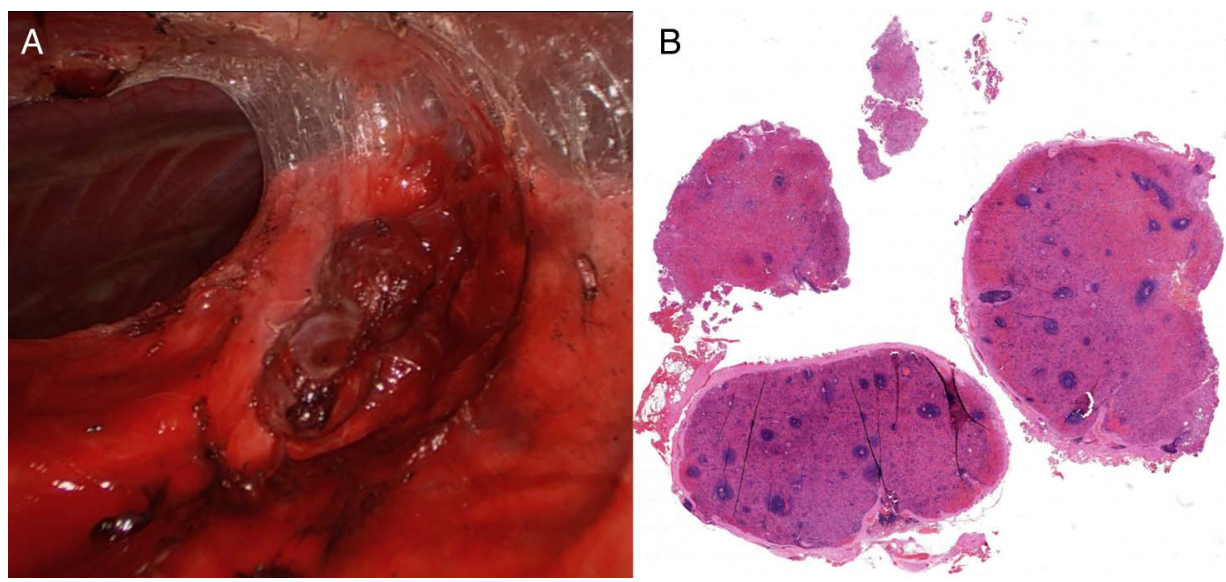

To cite: Remtulla $\mathrm{M}$

Figure 2 Multiple pleural nodules were found during video-assisted thoracoscopic surgery surrounded by adhesions in the lower left hemithorax $(A)$ and on microscopy, low power view of the excised lesions showed viable nodules of histologically normal encapsulated splenic tissue demonstrating red and white pulps (B). 


\section{Chest clinic}

the left kidney, adjacent to the splenic flexure and at the pancreatic tail.

The multidisciplinary team recommended biopsy of one of the nodules to establish a histological diagnosis. During video-assisted thoracoscopic surgery (VATS), the lower pleural space was obliterated by dense adhesions; after division of the adhesions and mobilisation of the lung, numerous dark brown, rounded lesions were seen scattered on the lung surface, diaphragm and mediastinal pleura, several of which were excised (figure 2A). Histology showed nodules of congested but largely unremarkable viable splenic tissue, confirming the diagnosis of splenosis (figure 2B); there was no malignancy.

Splenosis, the autotransplantation of splenic tissue following rupture or splenectomy, is rare, despite the relatively high incidence of splenic trauma. Ectopic splenic fragments implant onto a serosal surface, derive a local blood supply and develop into nodules of differentiated splenic tissue. It is most commonly found in the abdomen or pelvis, with seeding of the peritoneum, omentum or mesentery; few cases of thoracic splenosis associated with traumatic diaphragmatic rupture have been reported. ${ }^{1}$

Thoracic splenosis usually presents as an incidental finding several decades after splenic trauma and rarely causes symptoms. Pleural-based nodules may be mistaken for an intrathoracic malignancy, prompting invasive investigation with needle or VATS biopsy. If suspected, the diagnosis may also be made by
99m-Technetium heat-damaged erythrocyte scan, which has a high sensitivity and specificity for splenic tissue. ${ }^{2}$

Splenosis is a benign condition and does not require therapeutic excision. However, while the histology resembles normal splenic tissue, it is still associated with reduced immune function as the residual volume and function is insufficient to confer protection against overwhelming post-splenectomy infection, and therefore, the need for immunisation and early prophylactic penicillin remains. ${ }^{3}$

In conclusion, thoracic splenosis should be considered in a patient presenting with asymptomatic left pleural nodules and a remote history of thoracoabdominal trauma, especially in the setting of known splenic injury and diaphragmatic rupture.

Contributors All authors were involved in the management of the patient and composing the manuscript.

Competing interests None declared.

Patient consent Obtained.

Provenance and peer review Not commissioned; externally peer reviewed.

\section{REFERENCES}

1 Normand JP, Rioux M, Dumont M, et al. Thoracic splenosis after blunt trauma: frequency and imaging findings. Am J Roentgenol 1993;161:739-41.

2 Malik UF, Martin MR, Patel R, et al. Parenchymal thoracic splenosis: history and nuclear imaging without invasive procedures may provide diagnosis. J Clin Med Res 2010;2:180-4.

3 Connell NT, Brunner AM, Kerr CA, et al. Splenosis and sepsis: the born-again spleen provides poor protection. Virulence 2011;2:4-11. 